\title{
Power Quality Permanent Monitoring Systems in Romania
}

\author{
C. Stanescu ${ }^{1}$, J. Widmer ${ }^{2}$ and C. Pispiris ${ }^{1}$ \\ ${ }^{1}$ Romanian Power Grid Company TRANSELECTRICA \\ Armand Calinescu 2-4, Bucharest, Romania \\ Phone number: +40 269 207196, Fax number: +40 29 10928, e-mail: carmen.stanescu@transelectrica.ro, \\ cristian.pispiris@transelectrica.ro \\ ${ }^{2}$ Landis+Gyr Company \\ Feldstrasse 1, 6301 Zug, Switzerland \\ Phone number: +41 41935 6209, Fax number: +41 41935 6211, e-mail: jakob.widmer@landisgyr.com
}

\begin{abstract}
This paper addresses the results obtained from the first and second power quality permanent monitoring systems, implemented in the points of common coupling, between Transmission Operator and Supply Operator, Transmission Operator and Eligible Customers. Monthly statistic reports from these systems are received at the National Dispatch center and are included in the reports towards Romanian Electricity Regulatory Authority. The information gathered from these systems help to develop the Romanian PQ regulation, to verify the contractual requirements, to report the indices for network performance and to identify the solutions for network optimization, technical energy losses reduction and customer satisfaction.
\end{abstract}

\section{Key words}

Power Quality, Permanent Monitoring, System, Instrument, Point of Common Coupling.

\section{Introduction}

Transelectrica is the Romanian Transmission System Operator and at the same time, the Balancing Market and the Metering Operator of the wholesale electricity market. The power quality (PQ) aspects are very actual and important in this company activity at the interface with Electrica, the Distribution and Supply Operator, and with eligible customers. All the partners have the common goal of maintaining the PQ parameters in the points of common coupling (PCC), within admissible limits.

The PQ conditions in PCC between transmission and distribution power grid are regulated by The Electricity Transmission Grid - Standard of performance [1] and Technical Code [2]. At the same time, The Electricity Distribution Grid - Standard of performance [3] and Technical Code [4] are in force for distribution power grid. These were issued by Romanian Electricity Regulatory Authority (ANRE). The PQ conditions in PCC between transmission power grid and eligible customers are regulated by the Connection Notice issued by Transelectrica. In this regulation could be established PQ parameters admissible limits. These regulations define the quality of service as a combination of power supply reliability, supply quality and commercial quality, between the utility and the customers.

According to [1] Transelectrica has to monitor permanent or temporary in PCC and report monthly to ANRE the supply voltage quality regarding to: power frequency, magnitude, harmonic and unbalance.

1) Power frequency. - the admissible limits are defined in EU interconnecting systems conventions.

2) Magnitude of the supply voltage - the admissible limits for a nominal value of:

- $400 \mathrm{kV}$ are inside the $[380 \mathrm{kV} \ldots .420 \mathrm{kV}]$ interval;

- $\quad 220 \mathrm{kV}$ are inside the $[198 \mathrm{kV}$... 242kV] interval.;

- $110 \mathrm{kV}$ are inside the $[99 \mathrm{kV} \ldots 121 \mathrm{kV}]$ interval.

3) Voltage harmonics- the superior admissible limit for total harmonic distortion voltage is $3 \%$.

4) Supply voltage unbalance - unbalance is evaluated using the method of symmetrical components; and the superior admissible limit for negative sequence component is $1 \%$.

For this monitoring the equipments must be dedicated, with high accuracy and according with PQ international standards.

In order to achieve those goals, for two years, Transelectrica has developed:

1) Since 2005, dedicated CT and VT measuring transformers have been installed in each metering points of wholesale electricity market.

2) Since February 2006, the first PQ monitoring system has been dedicated to the permanent monitoring of the interface between transmission and distribution power grid, at a voltage level of $110 \mathrm{kV}$.

3) Since September 2007, the third PQ monitoring system has been dedicated to the permanent monitoring of the interface between the transmission power grid and 
the eligible customers, at voltage levels of $110 \mathrm{kV}$, $220 \mathrm{kV}, 400 \mathrm{kV}$.

\section{PQ monitoring systems}

\section{A. The first PQ permanent monitoring system}

In Transelectrica, for Sibiu Subsidiary the partners on wholesale electricity market can be easily located using the geographical map, see figure 1 and the energy transfer boundary, see figure 2 .

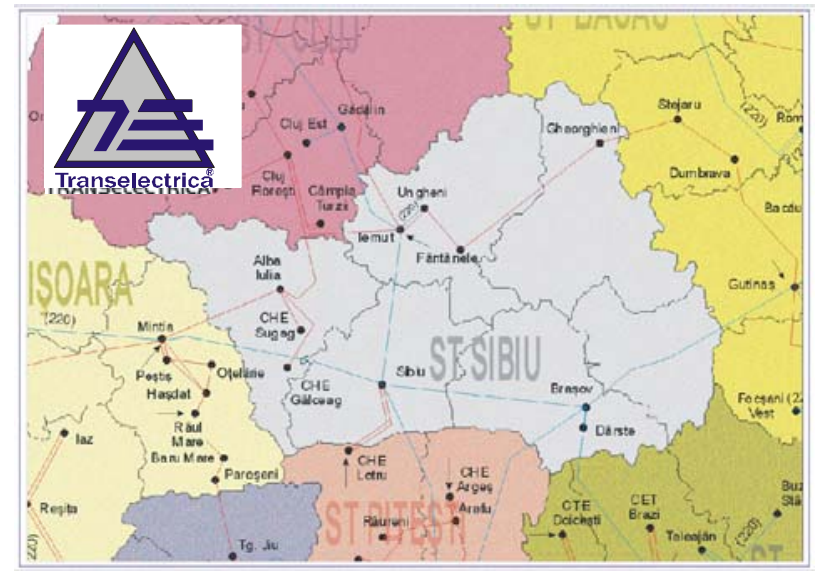

Fig. 1. Map of transmission network for Sibiu Subsidiary

Figure 2 contains all the partners and all the PCC, between transmission power grid and other systems, grouped according to substations site and voltage levels. PCC are very important to measure the quantity of the power received from, or delivered to others. Locations of installed PQ instrument are indicated using a blue circle.

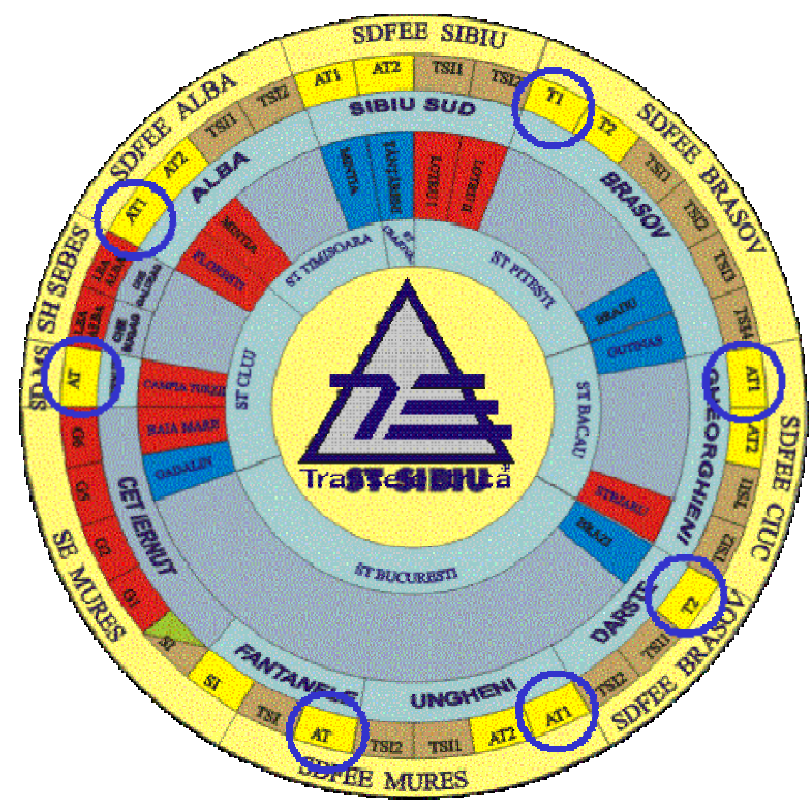

Fig. 2. Energy transfer boundary for Sibiu Subsidiary

The first PQ permanent monitoring system has been installed like a pilot, in the PCC, between transmission and distribution power grid. In each substation of Sibiu
Subsidiary was monitored one metering point at a voltage level of $110 \mathrm{kV}$ power auto/transformers.

The system architecture of the first PQ permanent monitoring system consists of:

- $\quad$ seven PQ instruments, type ION7650 [5], installed at substation level, inside seven Transelectrica's substations: Alba Iulia AT1/110kV, Brasov $\mathrm{T} 1 / 110 \mathrm{kV}$, Darste $\mathrm{T} 2 / 110 \mathrm{kV}$, Iernut $\mathrm{AT} / 110 \mathrm{kV}$, Fantanele AT/110kV, Gheorgheni AT1/110kV, Ungheni AT1/110kV;

- one database server, administrated by the software ION Management, installed at central level, at the headquarters of Transelectrica Sibiu Subsidiary, see figure 3 .

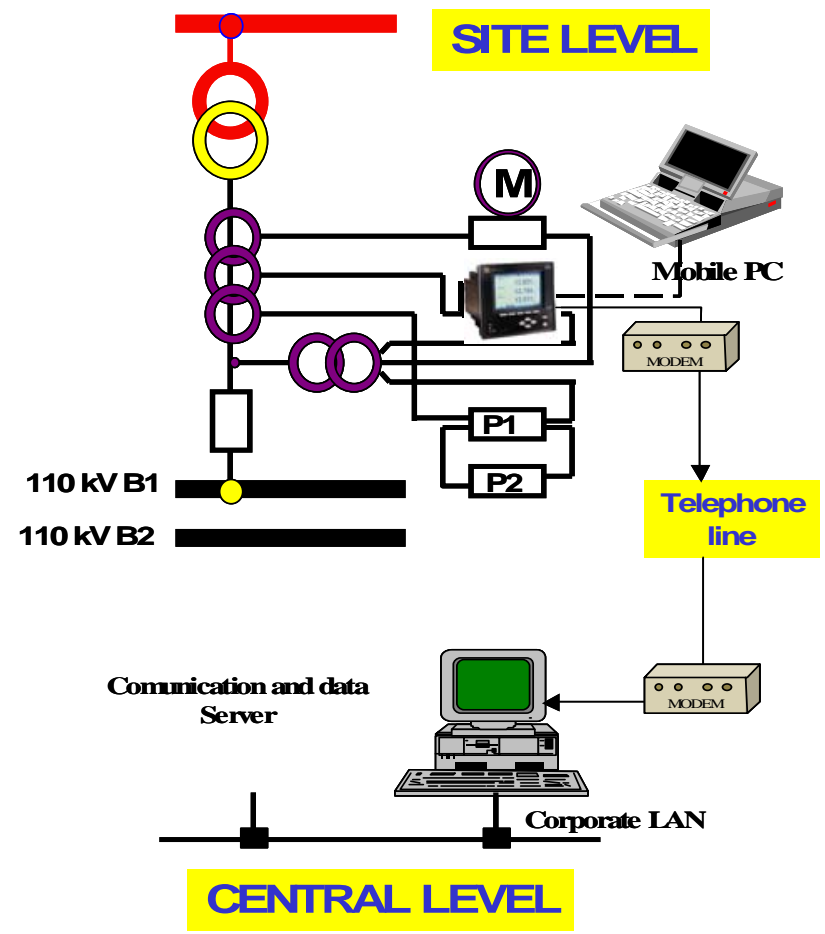

Fig. 3. The first PQ system architecture

The equipment used for this system has the functionality of a three phases static PQ instrument, disturbance analyzer and data logger in a single instrument. Fixed montage solution without current probes was adopted for the installation of PQ instruments in all seven locations. The PQ instruments are using 4 input currents and 4 input voltages, becoming part of the secondary winding with 0.2 accuracy class, from the dedicated measurement current and voltage transformers. The communication between site level and central level uses analogue modems, equipments for optical fiber medium, channel of $64 \mathrm{~kb} / \mathrm{s}$ digital link and standard protocol.

The data collected by PQ instruments are readout on request to the central level, stored in a common database and exported to Excel or HTML files. On central level ION Enterprise Management software configuration analyses all the PQ parameters of the transmission power grid and presents the EN 50160 statistics, with the admissible limits set for high voltage, according to [1]. 
Statistical reports, according with [7] are automatically generated for weekly determination of cumulative probabilities $95 \%$ of power frequency, supply voltage magnitude, supply voltage unbalanced, voltage harmonics, total harmonic voltage, interharmonic voltage, long time flicker variations according standard methods and limits [6].

Monthly the reports are sent to the National Dispatch and included in the reports towards ANRE. Also, the yearly reports of the supply voltage dips, overvoltages and interruptions are tabulate according to [7]. As an example, figure 4 presents a part of the report for Darste substation, in June 2007.

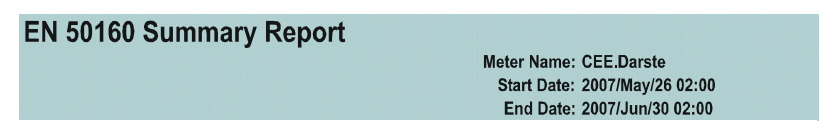

Complete Compliance in this Summary? №

Measurement and Observation Period Compliance Table
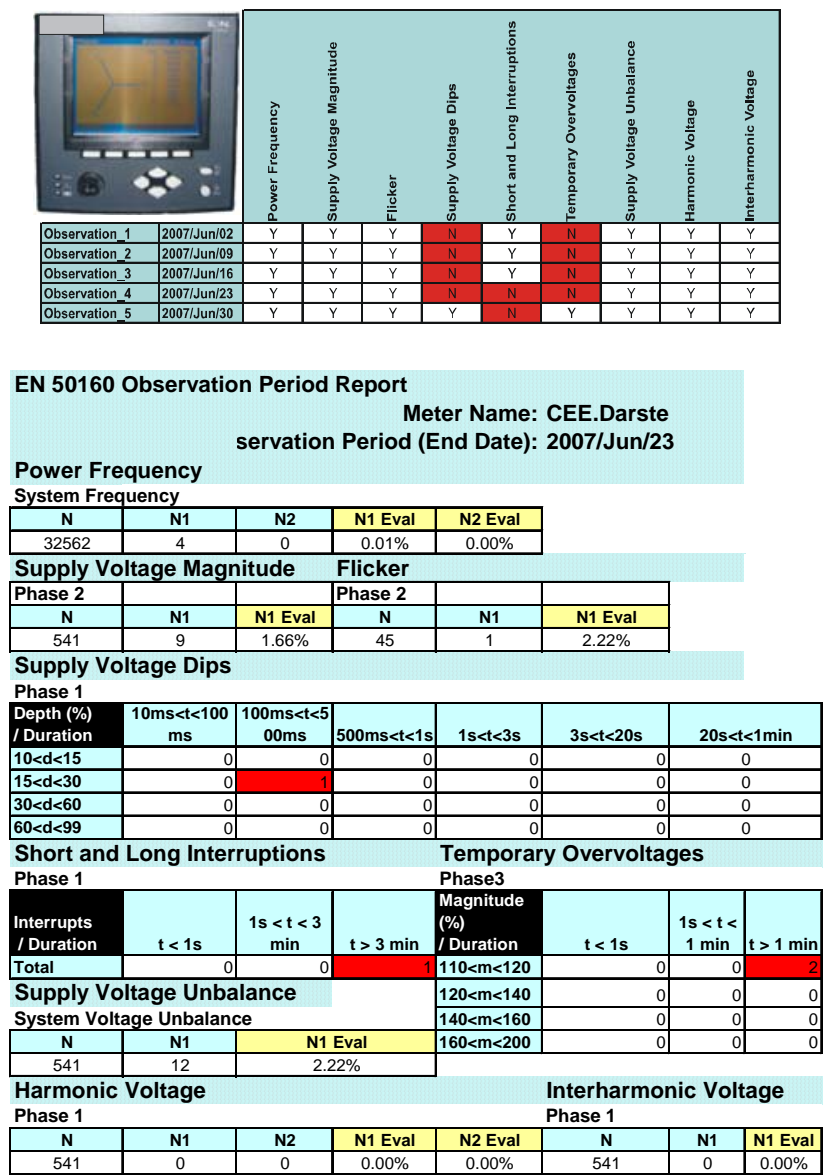

Fig. 4. The first PQ system reports

Because these PQ instruments are dedicated to PCC for wholesale energy market, during the year 2008 its have to be upgraded for class A performance and certification, according to [7].

\section{B. The second $P Q$ permanent monitoring system}

The wholesale energy market in Romania has been $100 \%$ liberalized since 01.07.2007 and from September 2007
Transelectrica had implemented a permanent monitoring system at the interface between transmission power grid and all eligible customers supplied by transmission power grid, according to a dedicated study [11].

Special attention is required for customers supplied at very high voltage levels: COST Targoviste, the biggest plant in metallurgy industry, ALRO, the largest aluminum smelter in Central and Eastern Europe, DUCTIL Steel , Iron Plant Resita and MECHEL Campia Turzii focused on mining products, rolled steel, casting products.

The system architecture of the second PQ permanent monitoring system consists of:

- fifteen PQ instruments, type ZMQ202, very high precision meter with dedicated PQ recorder module [9], installed at substation level, inside eight Transelectrica's substations: Otelarie 220kV, Resita $220 \mathrm{kV}$, Iaz $220 \mathrm{kV}$, Slatina $220 \mathrm{kV}$, Targoviste 220kV, Campia Turzii 220kV, Roman Nord 220kV, Tulcea Vest 400kV;

- one database server, installed at central level, at the headquarters of Transelectrica OMEPA Sibiu Subsidiary, see figure 5. The main communication between site level and central level uses optical fiber medium and GSM modems, for backup.

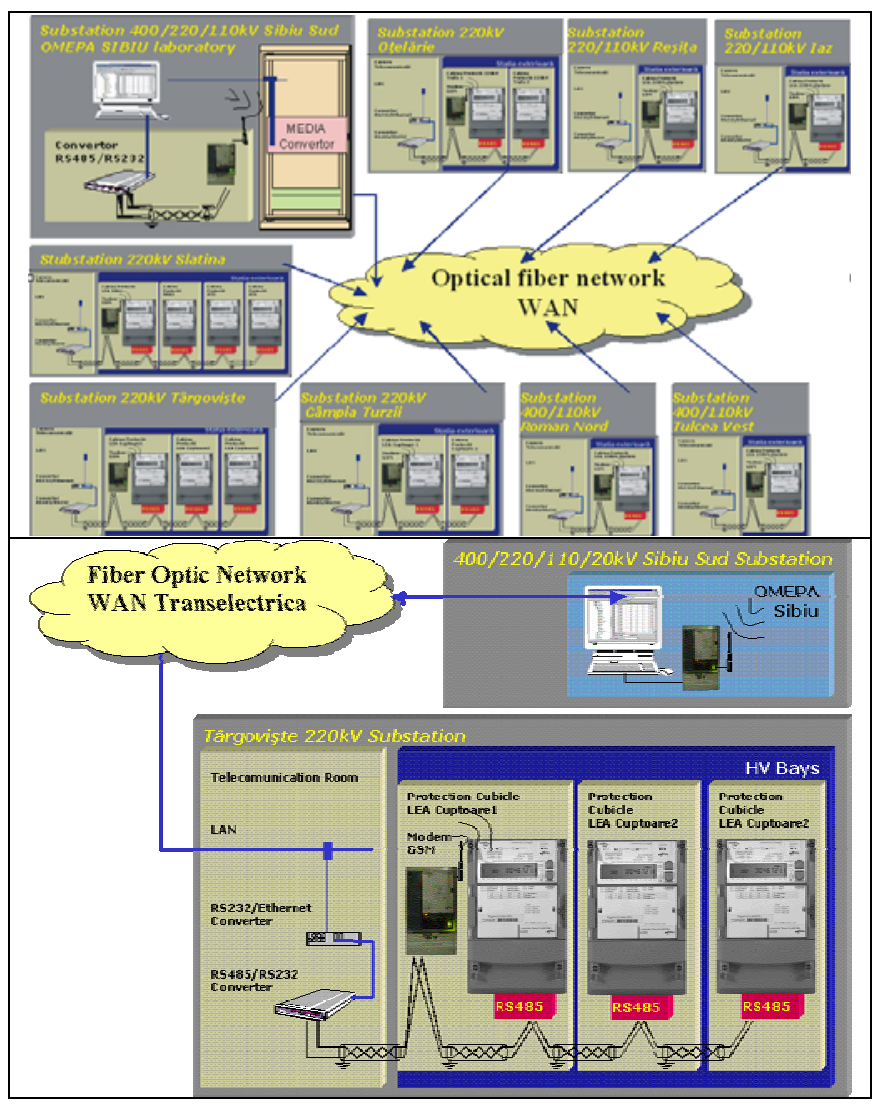

Fig. 5. The second PQ system architecture

Fixed montage solution without current probes was adopted for the installation of PQ instruments. The advantages in using high precision instruments with $P Q$ 
recorder instead of separate meter and separate PQ instrument are:

1) reduced cost of installation;

2) access to the accuracy class $0.2 \mathrm{VT}$ and CT without additional wiring, no additional burden for CT;

3) only one device on customers side;

4) separate channels for billing data and PQ-data.

The parameterization, measured value acquisition, event analysis and reporting are managed by Simeas Q PAR, Sicaro Q Manager and Sicaro PQ software [10]. 'Statistical analysis of network quality' reports according to [7], for admissible limits according to [1] are issued automatically for weekly analyse of cumulative probabilities 95\%, maximum, minimum, and average values. Monthly these reports are sent to the National Dispatch. The reports are generated like an overview, presenting numerical and graphical analyses of the PQ parameters: power frequency, supply voltage magnitude, supply voltage unbalanced, total harmonic voltage, voltage harmonics, current harmonics, flicker $\mathrm{P}_{\mathrm{lt}}$, slow voltage changes. All are exported to HTML files, with links for each PQ parameter, as shown in figure 6 .

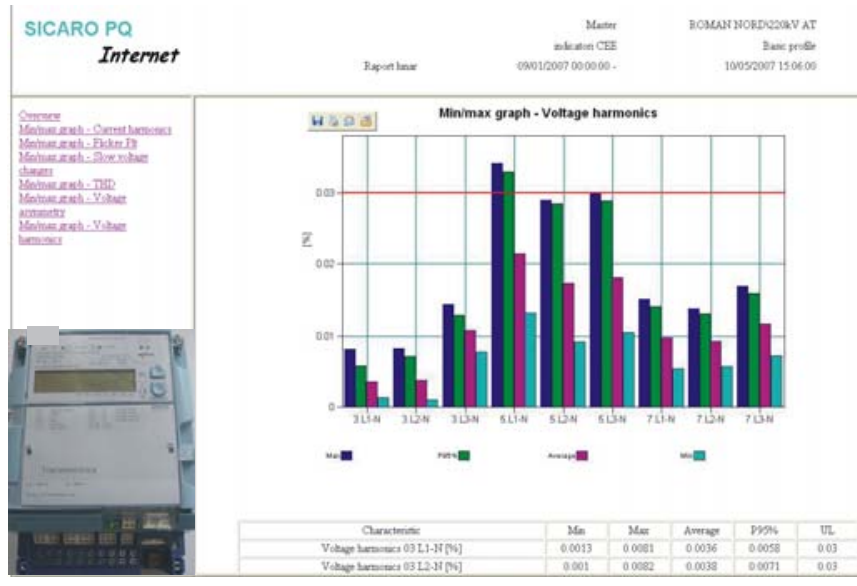

Fig. 6. The second PQ system report

Also very useful features from PQ instruments are: to record the outages, under/over voltage with selectable thresholds, voltage dip table with duration and depth. These software applications are very user friendly and flexible in order to set the graphic system structure, parameterize the measurement settings, transfer and store measured data, and export and store data in ASCII format.

\section{Further Information}

Monthly statistic reports from PQ permanent monitoring systems are received at the National Dispatch center and are used to prepare the report for ANRE, according to [1]. Further we propose future developments such as integration of the reports generated by the systems, which will provide access for different users like National Dispatch, via internet browsers (figure 7).

The goal of this integrated system is to assist the dispatcher in taking the necessary action to keep the PQ parameters in admissible limits, adopt the configuration for single line diagram, or the connection of different voltage tap of autotransformer. With Transelectrica investment during the year 2008 a software platform will be developed to integrate these systems.

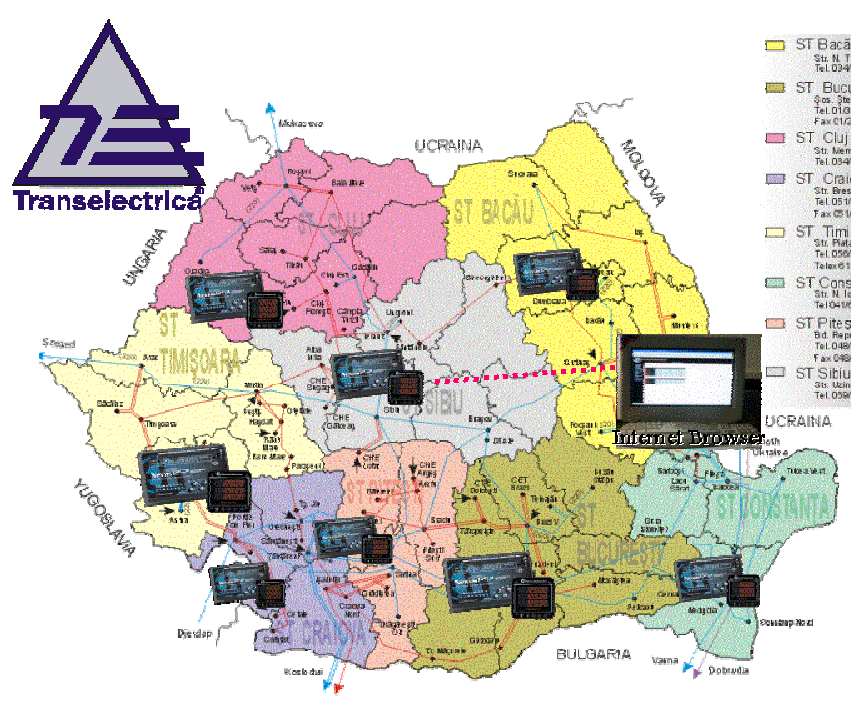

Fig. 7. The future of the PQ permanent monitoring systems

\section{Conclusion}

World wide exists the need to create standards for the measurement, analysis and validation methods for all PQ parameters for $\mathrm{HV}$. In Romania, in order to create a unitary system, the performance standards for transmission and distribution power grid need to be correlated. The scope is to define clear and complete the performance $\mathrm{PQ}$ parameters at the interface between transmission and distribution power grids and in the PCC, the specific duties of each power grid operator and those of the users connected to the power grid. Continuous PQ monitoring systems are the necessary steps towards the development of PQ regulation.

On the wholesale energy market, the monitoring of the PQ parameters requires the use of class A performance PQ instruments. Also, the equipments/software needs to be used in PQ analysis together with a good knowledge of the measurement uncertainty, calculation formulas, and the implemented measurement, aggregation and ascertainment methods. World wide, the PQ dynamic requests continuous information in order to upgrade the existing instruments, or to replace them with new one in order to respect the new PQ standards. In this field there is a need for qualified personnel. The dedicated training classes Leonardo Power Quality Initiative Vocational Education System are forming a large number of Romanian experts starting with $1^{\text {st }}$ level experts in 2006 and continuing with $2^{\text {nd }}$ level experts in 2008.

The permanent monitoring of power quality parameters is used to verify the contractual requirements presented in regulations. Special attention is required for customers connected at very high voltage (steel and aluminum processing plants). Perturbations generated by their 
technological processes are transmitted over transmission power grid and affect PQ parameters, for other customers located at great distance about the PCC, like the voltage harmonics and flicker.

At the same time PQ has an important effect regarding network economic efficiency and represents a defining parameter for performance network evaluation. The PQ permanent monitoring systems presented above are used to monitor the performance evolution of a network during long periods, to compare different networks evolutions during the same period. Hereby we propose to extend the permanent PQ monitoring by implementing new systems that will optimize the power grid operating mode for network optimization, technical energy losses reduction and customer satisfaction.

\section{References}

[1] ANRE, Romania 2007, "The Electricity Transmission Grid - Standard of performance”, www.anre.ro

[2] ANRE, Romania, 2004, "The Electricity Transmission Grid - Technical Code”

[3] ANRE, Romania, 2007, "The Electricity Distribution Grid - Standard of performance"

[4] ANRE, Romania, 2000, "The Electricity Distribution Grid - Technical Code”

[5] Power Measurement, Canada, "Technical documentation for ION Meters 7650”, www.pwrm.com

[6] IEC 61000-3-7, 1996 EMC part 3-Limits, Section7: "Assessment of emission limits for fluctuating loads in medium and high voltage power systems".

[7] SR EN 50160, Romania, 1998, "The Characteristics of The Voltage in Romanian Public Distribution System

[8] IEC 61000-4-30, 2003, EMC part 4-30 “Testing and measurement techniques - Power Quality Measurement Methods"

[9] Landis+Gyr, Switzerland, "User manual for high precision metering ZMQ 202”, www.landisgyr.com

[10] Siemens, Germany, 'Configuration software for SIMEAS Q’, www.powerquality.de

[11] Institute Study Design Engineering, Romania, 2006, the study "Power Quality Analyzing System at the eligible customers” 
